\title{
Evaluated Stress and Anxiety in College Students before and After Midterm Exam
}

\author{
Rozy Kumari ${ }^{1}$,Monika Sharma ${ }^{2}$, Ambreen Fatima ${ }^{2}$, \\ Pramod Kumar Sahu' ${ }^{2}$, Shahiduz Zafar ${ }^{3}$, Samreen Fatima ${ }^{4}$ \\ ${ }^{1} B P T 4^{\text {th }}$ Year Student, Galgotias University, Greater Noida, ${ }^{2}$ Assistant Professor, Galgotias University, Greater \\ Noida, ${ }^{3}$ Professor, Galgotias University, Greater Noida, ${ }^{4}$ Phd. Scholar, AMU, Aligarh, U.P.
}

\begin{abstract}
The purpose of this study is to assess anxiety and stress in college student before and after midterm exam. The study will be conducted on N=200 students of Galgotias University, Greater Noida, U.P., India. Scale is taken as outcome measure and students are asked to fill a form (according to DASS and PSS) after taking consent before and after midterm examination. There collected data is then to be compared to know the effect of exams on stress and anxiety level of students.

Background: The spiritual well-being of university student is an area of amplify reflection universal. The aim of this student is to check-up the expansion of stress and concern into a group of Galgotias university student. Solely as each young person, undergraduate students needfulness to cope.

Method: We are taking 200 subjects for this study. My study is based on observational study. We are using DASS and PSS scale for measuring the stress and anxiety of the students. Inclusion criteria is stress and anxiety in college student during midterm exam and exclusion criteria is neurological and psychological disorders.
\end{abstract}

Results: We are hoping for positive result. We would find that the students take exam stress and anxiety level increase.

Discussion AND Conclusion: We would conclude positive result of our study. Our study will show that during and before exam students face stress and anxiety and after exam it will decrease.

Keywords: Stress, anxiety, midterm exam, scale.

\section{Introduction}

Stress: Stress is an inescapable sensation in every phase of human life. In a medical or biological context stress is a physical, mental, or emotional factor that causes bodily or mental tension. Stresses can be external (from the environment, psychological, or social situations) or internal (illness, or from a medical procedure. ${ }^{1}$

\section{Corresponding Author:}

Rozy Kumari

Student (BPT), Greater Noida

e-mail: rozy.kumari@galgotiasuniversity.edu.in
Anxiety: Depression and anxiety are the most common types of mental disorders. Many individuals with anxiety even have depression and the other way around. Anxiety and depression have been found to be more widespread among college students than the general masses. $^{2}$

DASS scale: The DASS is a self-administered device with well-functioning psychometric properties in community and clinical samples, and has been described to differentiate between the three states of depression, anxiety and stress.

The depression scale is mainly to assess hopelessness, dysphoria, self-deprecation,devaluation of life, lack of interest or involvement. 
The anxiety scale can also measure autonomic arousal, striated muscle effects, situational anxiety and subjective experience on anxious effects.

The stress scale is affected by the measure of chronic non-specific arousal. The scale assesses difficulty relaxing, nervous arousal and being easily upset or agitated, irritability or over-reaction and impatience. ${ }^{3}$

PSS scale: The "PSS" suggested by Cohen et al. in 1983 measures the degree to which individual situations are evaluated as being stressful or more precisely, unpredictable, uncontrollable and intense.

Many authors (Cohen, 1978; Averill, 1973; Lazarus, 1966) only mentioned these three main categories intensity, uncontrollability and unpredictability, are the most vulnerable components of the experience of stress, which are usually measured by objective-stress scales. ${ }^{4}$

Exam anxiety may be a set of responses that has excessive worry, depression, nervousness and irrelevant thinking which is experienced by many students while undertaking any exam. There are four main areas of reported stresses which can definitely lead to anxiety of exam -

(a) Issues of life style,

(b) Decrement of required information,

(c) Style of studying and

(d) Psychological factors.

Life styles issue basically include improper rest, lack of time, poor nutrition and insufficient physical activity management leading to exam anxiety as reported by many authors. ${ }^{5}$

Issues that contribute to their stress and anxiety include academic challenges, clinical challenges, technological advances, financial concerns, interpersonal difficulties, family problems, physical and mental health issues, inadequate support, and poor coping skills. Additional stressors include cultural adjustments, language issues, social isolation, and discrimination. Both stress and anxiety are universal among students. ${ }^{6}$ Anxiety and depression commonly co-occur. This overlapping can be seen at the level of anxious and depressive mood, symptoms, and disorder from samples of children through adults.

It is a fact that emotional symptoms and disorders overlap which leads to difficulties in testing causal models for either depression or anxiety.

Given the pattern of high overlapping between anxiety and depression, a researcher cannot be certain whether a assumed causal factor for specific symptoms (e.g., depression) is, in fact, contributing to those particular symptoms unless both anxiety and depression are assessed properly.

Few studies in the literature have tested causal models of anxiety or depression while measuring both anxiety and depressive symptoms to ensure that the proposed etiological factors are affectively specific as hypothesized. The primary aim of this study has been to test whether mental weakness for depression inter-acts with negative life event stop or reduce depression more specifically compared with anxiety. ${ }^{7}$

Numerous studies on exam stress have shown that exam periods and exam situations are stressful for students. Some of the studies showed an increase in anxiety level, depressiveness and negative emotional states.

It has been observed that there is an increase in bad mood, lack of energy, bodily discomfort, somatic strain and restlessness of students during pre-exam period and even their cortisol level and heart rate were increased after exams. ${ }^{8}$ The mechanisms of stress-related immune changes have not been fully uncovered. Cell-mediated immune responses as well as antibody and certain cytokines are reported as being suppressed during times of high stress. ${ }^{9}$ These studies indicate that academic exam stress may pose an effective model for the study of stress- related to sleeping problems with high external validity. ${ }^{10}$

Stress, at its simplest, is defined as the magnitude to which life circumstances are considered worrying. Crosssectional study focuses on the relationship between supposed stresses. In this study, levels of perceived stress showed a significant association between the frequency of health complaints and symptoms and levels of psychological symptoms, such as depression, mood swings, and anxiety.

Emotion-focused strategies are emotionally driven, while avoidance coping strategies are those that seek distraction or removal from the stress- producing situation. In general, greater reliance on avoidance and emotion-focused coping and a lack of problem or taskfocused coping has been associated with poor emotional 
adjustment, psychosocial dysfunction, low self-esteem, and higher levels of anxiety, depression, apathy, and denial. ${ }^{11}$

Anxiety is associated with an increase in catecholamine's which is responsible for an increase in magnesium urinary excretion and a decrease in its plasmatic concentrations. The notice able increase in muscular tension linked to anxiety consumes an important amount of energy that is partially due to the ATP-AD transformation. A high increase in urinary phosphate excretional so contributes to the magnesium reduction. Indeed, magnesium has been proposed for treatment in different anxiety disorders. ${ }^{12}$ The effectiveness of exam stress as a model of psychosocial stress has repeatedly been shown on immunological neuroendocrine, physiological and psychological parameters. Despite these associations, exam stress has not been used to investigate predisposing factors of Somatization so far Determined the effects of exam stress on Somatization, showing a significant positive relationship in 38 participants. Still, no quantitative description of Somatization symptoms under exam stress is available, although the somatic symptoms of acute exam anxiety have been assessed systematically. Expected to increase under exam stress and return to baseline after a period without exam. According to the stress-alexithymia hypothesis, and show a stronger association with these increases than neuroticism, state anxiety, or depression. ${ }^{13}$ According to the Anxiety Disorders Association of America (2012), anxiety disorders are the most commonly diagnosed of mental health conditions with more than 40million Americans affected annually. College students are no exception to these trends. In a recent study conducted by the American College Health Association (2009), stress ranked as the number one health issue that impedes students' academic performance. In addition, the latest annual survey of college counselling centredirectors across the country indicated anxiety and depression as the top two presenting concerns for their student clients(Association for University and College Counselling Center Directors [AUCCCD],2011). ${ }^{14}$ In addition to anxiety and stress, depression can also impact college life to such an extent that in-depth research is necessary in order to help future students. ${ }^{15}$

\section{Methodology}

The purpose of this study was to examine the impact that depression and anxiety have on academic performance among college students, during midterm exam. This study was designed to decipher whether students with anxiety and depression symptoms may prevent lower academic performance during before and after midterm exam. This is a cross-sectional study.All the students were selected from Galgotias University from various courses were considering as population of the study. The students were chosen randomly from Galgotia's university at greater Noida. Total numbers of students are 100

\section{Instrument:}

- DASS SCALE: The DASS is a self-administered instrument with well-ordered psychometric properties in clinical and community samples, and has been shown to differentiate between the three states of depression; stress and anxiety. The Turkish version of DASS was constructed by Nuran Bayram et al. ${ }^{3}$ and used in a study of a non-clinical sample. The reliability coefficients (Cronbach's alpha) of the Turkish version of DASS-42 in our study were found for depression, stress and anxiety to be $89.1,82.6$ and $85.9 \%$, respectively.

- PSS SCALE: The Perceived stress scale (PSS) is the most widely used psychological instrument for measuring the perception of stress.

This randomized controlled study was conducted with college students. The study was conducted at Galgotia's University. This is a mixed sample $(n=100)$ of a population ofuniversity students made up of 53 female (53\%) and 47 male $(47 \%)$ for before midterm exam. This survey aimed at defining the students to evaluated stress and anxiety in college students before and after midterm exam.

Research Design: Data was collected using a offline questionnaire survey form. The requirements to participate include being college students from various branches and having been enrolled at the university in fall of 2020. The surveys were administered offline in conjunction with an informed consent form with survey form. Both the scales (DASS \& PSS) questionnaire form distribute to college students to collect data and after filling the form collect from students. Data analysis is done on the basis of both scales.

\section{Result Analysis}

Demographic value analysed by Independent $\mathrm{T}$ test for Age. 
Table No 1 demographic value of age is analysed by the $T$ test

\begin{tabular}{|l|c|c|c|c|}
\hline & Before Exam DASS N= 100 & After Exam DASS N=100 & T Value & Level of Significance $\mathbf{P}$ \\
\hline Average Age & $23.6 \pm 3.17$ & $23.6 \pm 3.17$ & $\mathrm{O}$ & 0.5 Non significance \\
\hline
\end{tabular}

Table No 2. Comparative study of DASS before and after exam by one way ANOVA analysis

\begin{tabular}{|l|c|c|c|}
\hline & Before Exam DASS & After Exam DASS & Level of Significance P Value \\
\hline Comparative Study & $60.81 \pm 22.98$ & $1.08 \pm 1.16$ & $0.001^{*}$ \\
\hline
\end{tabular}

- Table No 2 the comparative study shows that there is decrease DASS average after the DASS exam before it was 60.81 and standard deviation is 22.89 and the DASS after the exam was 1.08 ad stand deviation is 1.16 respectively.

Table No 3: Demographic data of age is evaluated by independent $T$ test

\begin{tabular}{|l|c|c|c|c|}
\hline & Before PSS N=100 & After PSS N= 100 & T Value & Level of Significance P value \\
\hline Average Age & $23.6 \pm 3.17$ & $23.6 \pm 3.17$ & 0 & 0.5 non significance \\
\hline
\end{tabular}

Table No 4: Comparative study of PSS before and after by the one way ANOVA.

\begin{tabular}{|l|c|c|c|}
\hline & Before PSS Average & After PSS Average & Level of significance P value \\
\hline Comparative study & $18.39 \pm 10.01$ & $0.56 \pm 0.55$ & $0.001 *$ \\
\hline
\end{tabular}

- The table no 4 shows that the Average PSS value before was 18.39 with standard deviation $10.01 \&$ average after PSS was 0.56 and standard deviation is 0.55 , the result that there is great decrease of PSS after the exam.

\section{Discussion}

Anxiety is one if the biggest and dangerous hunter of academic performance (Mc Craty, 2007 and McCarty, et al., 2000) and various studies have clearly showed that it shows a detrimental effect. Thus it is clear that there is a possible relation between high level of anxiety and low academic performance in students. Researchers made it clear that high levels of anxiety results in the decrease of, distraction, working memory and reasoning in students (Aronen et al., 2005). Researchers have been searching for the relation between anxiety and its effect on academic performance among school students. The Students suffering from high level of anxiety have a less memory span; less concentration, and lack of confidence, and a devastating reasoning power. Generally, high level of anxiety was more closely associated with lower performance among low ability students. ${ }^{1}$ It is important to note that the population for this study is all SOAR students who were selected in the fall of 2016, not the population of all ACU students. And no data for SOAR Population is available; therefore the statistics of the population of ACU students during the Fall of 2016 semester were examined. In doing so, this may also reveal certain characteristics that are specific to the SOAR population. ${ }^{2} \mathrm{We}$ found that our sample of students had higher mean depression, anxiety and stress scores compared with previously published normative data. Although the DASS is not diagnostic instrument, it is worth remembering that the rates of depression, anxiety and stress symptoms of moderate severity or above may require attention from health- care professionals. In our study, the distributions of students $(N=200)$ regarding depression, anxiety and stress symptoms of moderate severity or above were $27.1,47.1$ and $27.0 \%$, respectively. Previousdata on gender difference in depression, anxiety and stress scores from DASS were inconclusive.$^{3}$

This exploratory method made it possible to show a "medium zone" which does not fall within the transactional logic of the theory of stress defined as a "discrepancy" between the primary assessment of the threat and the secondary assessment elated to the resources. Thus, how does one consider an averagely stressed individual who would have a high score of both perceived distress and perceived control according to the PSS tool . ${ }^{4}$ The anxiety of exam is the emotional outcome 
that some students have to face before the exams. The fear is not irrational, but excessive fear interferes with performance. Many researchers suggest that a little worry is good for students because it keeps them task oriented; however excessive worry on the other hand can be very debilitating and interferes with the results if not managed appropriately Psychological factors including irrational thoughts about exams and result, negative thinking, selfcriticism and feeling of no control over exam situation were reported by sixty to sixty-five percent students. The results of the study show extensive course load and long duration of exams as the major contributors to exam anxiety . ${ }^{5}$ Based on the findings from previous studies, both anxiety and stress levels are expected to boom for nursing students when they start their first clinical training and if they do not receive any interventions. The results from this study demonstrate that the 5-week biofeedback training intervention not only monitored the nursing students' stress levels from increasing, but also significantly reduced their levels of anxiety, a follow-up study on the impact of the biofeedback training on stress, anxiety, and academic performance of nursing students after one to two years will help nurse educators to better understand the long-term efficacy of the biofeedback intervention program .6 This etiological affective specificity was found regardless of whether composite depression and anxiety were used or whether the specific tripartite theory measures of an hedonic depression and anxious arousal were examined. Negative life events operated as a nonspecific risk factor for both depression and anxiety as prospective changes in stressors over time were associated with elevations in affective symptoms. The cognitive vulnerability-stress interaction was found to predict depression. Showed that neither cognitive vulnerability-stress inter- action was left significant after controlling for the other model's of vulnerability-stress component. ${ }^{7}$

In particular, total sleep time, subjective sleep quality, and daytime sleepiness have been associated with academic performance although parameters like morning-ness evening- ness and early late bedtimes have also been shown to play a role. In the present study we found that students reported to spend significantly less time in bed during the exam period, 0.5 hours less on average this reduction suggests that students alter their sleeping schedule while studying for exams. ${ }^{10}$ To my knowledge, this is the first study to look at the psychosocial factors that impact negatively on chiropractic students and factors that may be protective during their education. As such, it could impact on the findings finally, this survey was cross-sectional in nature. Causality between the coping strategies, resilience, and overall health dimensions cannot be assumed. A longitudinal study following the students over the length of the program may add further insight in the relationships between these factors. ${ }^{11}$ The present results showed that the evaluated students did not experiment a stress increase during exams but suffered a significant anxiety increase. It is interesting to observe that the psychological findings agree with urinary biomarkers studied. It is known that anxiety is related to partial magnesium decrease associated with an increase in urinary magnesium excretion. ${ }^{12}$ The results of this study found that the group of participants who received biofeedback training in conjunction with counselling reported significantly greater reduction in anxiety symptoms than did the group of participants who received counselling alone. One unique aspect of the study is that it was conducted within a college counselling centre and participants were recruited from the actual pool of students seeking counselling services. Given the number of students seeking mental health services for anxiety and stress-related issues, this study may provide a rational for greater implementation and all location of biofeedback training services to be provided within college counselling centres. ${ }^{14}$ While one of the initial goals of this survey was to narrow down possible correlates of depression, anxiety, and stress, all the potential sources of concern indicated on the survey had a significant positive correlation with levels of depression, anxiety, and stress. Of the 19 sources of concern surveyed, the 10 that caused the most concern were academic performance, pressure to succeed, postgraduation plans, financial concerns, quality of sleep, relationship with family relationship with friends, overall health, body care, and self-esteem. When the scores for anxiety, depression, and stress were matched to living standard, the students who used to live offcampus were the most stressed, anxious, and depressed ones. In addition, to transfer students scored the highest marks in the three areas measured by the DASS, with a significant difference in anxiety levels between transfer and non-transfer students. Lastly, upperclassmen scored the highest on the depression, anxiety, and stress scales when compared with underclassmen. ${ }^{15}$

\section{Conclusion}

This study concluded that on the basis of comparative values DASS \& PSS after and before midterm exam, it 
shows the decreased level in anxiety and depression after the midterm exam so it is finally concluded that there is presence of depression and anxiety during the students before the exam and it gets decreased automatically after midterm exam.

\section{Funding Sources: Self}

\section{Conflict of Interest: NA}

Ethical Clearance: Institutional ethical committee

\section{Reference}

1. Pathak HS, Mandal T, Mondal T, Das PP, Chakraborty U, Paria TK, Biswas P. A Retrospective descriptive study of snake bites in the medicine wards of Alipurduar hospital, West Bengal, India, from April 2013 to March 2014. J Indian Med Assoc. 2020;118(1):11-6.

2. Bisson KH. The effect of anxiety and depression on college students' academic performance: Exploring social support as a moderator.

3. Bayram N, Bilgel N. The prevalence and sociodemographic correlations of depression, anxiety and stress among a group of university students. Social psychiatry and psychiatric epidemiology. 2008 Aug 1;43(8):667-72.

4. Cerclé A, Gadea C, Hartmann A, Lourel M. Typological and factor analysis of the perceived stress measure by using the PSS scale. European review of applied psychology. 2008 Dec 1;58(4):227-39.

5. Hashmat S, Hashmat M, Amanullah F, Aziz S. Factors causing exam anxiety in medical students. Journal-Pakistan Medical Association. 2008 Apr 1;58(4):167.

6. Ratanasiripong P, Ratanasiripong N, Kathalae D. Biofeedback intervention for stress and anxiety among nursing students: a randomized controlled trial. International Scholarly Research Notices. 2012;2012.

7. Hankin BL, Abramson LY, Miller N, Haeffel
GJ. Cognitive vulnerability-stress theories of depression: Examining affective specificity in the prediction of depression versus anxiety in three prospective studies. Cognitive therapy and research. 2004 Jun 1;28(3):309-45.

8. Šimić N, Manenica I. Exam experience and some reactions to exam stress. Human physiology. 2012 Jan 1;38(1):67-72.

9. Marshall Jr GD, Agarwal SK, Lloyd C, Cohen L, Henninger EM, Morris GJ. Cytokine dysregulation associated with exam stress in healthy medical students. Brain, behavior, and immunity. 1998 Dec 1;12(4):297-307.

10. Zunhammer M, Eichhammer P, Busch V. Sleep quality during exam stress: the role of alcohol, caffeine and nicotine. PloS one. 2014 Oct 3;9(10):e109490.

11. Innes SI. The relationship between levels of resilience and coping styles in chiropractic students and perceived levels of stress and wellbeing. Journal of Chiropractic Education. 2017 Mar;31(1):1-7.

12. Grases G, Pérez-Castelló JA, Sanchis P, Casero A, Perelló J, Isern B, Rigo E, Grases F. Anxiety and stress among science students. Study of calcium and magnesium alterations. Magnesium research. 2006 Jun 1;19(2):102-6.

13. Zunhammer M, Eberle H, Eichhammer P, Busch V. Somatic symptoms evoked by exam stress in university students: the role of alexithymia, neuroticism, anxiety and depression. PloS one. 2013 Dec 18;8(12):e84911.

14. Paul Ratanasiripong, Kevin Sverduk, Judy Prince, Diane Hayashino,Biofeedback and Counseling for Stress and Anxiety Among College Students: https://doi.org/10.1353/csd.2012.0070

15. Beiter R, Nash R, McCrady M, Rhoades D, Linscomb M, Clarahan M, Sammut S. The prevalence and correlates of depression, anxiety, and stress in a sample of college students. Journal of affective disorders. 2015 Mar 1;173:90-6. 